\title{
Kinetic research on heterogeneously catalysed processes: A questionnaire on the state-of-the-art in industry
}

\author{
A.N.R. Bos ${ }^{\mathrm{a}}$, L. Lefferts ${ }^{\mathrm{b}}$, G.B. Marin ${ }^{\mathrm{c}, *}$, M.H.G.M. Steijns ${ }^{\mathrm{d}}$ \\ ${ }^{4}$ Shell Nederland Chemie. P.O. Box 3005, 3190 GB Hoogvliet. The Netherlands \\ ${ }^{\mathrm{b}}$ DSM. P.O. Box 18, $6160 \mathrm{MD}$ Geleen. The Netherlands \\ 'University of Gent. Laboratorium voor Petrochemische Techniek, Krijgslaan 218, B-9000 Gent. Belgium \\ ${ }^{\mathrm{d} D o w}$ Benelux, P.O. Box 48, 4530 AA Terneuzen. The Netherlands
}

\begin{abstract}
On the initiative of the Working Party 'Chemical Engineering in the Applications of Catalysis' of the European Federation of Chemical Engineering an assessment of the issues in the determination and application of kinetic data within the European industry was performed. The basis of the analysis consisted of a questionnaire put together by researchers from Dow, DSM. Shell and Eindhoven University of Technology. The 24 companies. which have responded to the questionnaire. can be classified into four groups: chemical, oil, engineering contractors and catalyst manufacturers. From the overall input it appears that there are three, equally important, utilisation areas for kinetic data: process development, process optimisation and catalyst development. There is a wide variety of kinetic data sources. Most of the respondents make use of test units which were primarily designed for development and optimisation. Avoiding transport limitations is, certainly in the case of short range projects or for complex feedstocks, not always taken care of. With respect to the modelling approaches, a common philosophy is 'as simple as possible'. Most of the respondents state that 'in principle' one should strive for intrinsic kinetics. but the majority nevertheless does for various reasons not separate all transport phenomena from reaction kinetics. Kinetic models are mostly simple first or $n$th order or Langmuir-Hinshelwood type expressions. More complex kinelic models are scarcely used. Three areas were frequently identified to offer opportunities for improvement. Gathering of kinetic data is too costly and time consuming. There is no systematic approach at all for determination and application of kinetics in case of unstable catalytic performance. Furthermore, the software available for the regression of kinetic data to rate equations based on mechanistic schemes as well as software to model reactors are insufficiently user friendly. The majority of the respondents state that the problems indicated should be solved by cooperation, e.g. between companies, between industry and academia and between the catalysis and the chemical engineering community. A workshop on the above topics was held in December 1996 with 15 companies and 6 academics attending. More information can be obtained from the secretariat of the Working Party.
\end{abstract}

Keywords: Kinetics; Reaction engineering; Modelling; Experimental techniques; Rate equations

\section{Introduction}

Thirty seven researchers within mainly European (petro-)chemical companies, catalyst companies and

*Corresponding author. contractors were asked for their opinion in July 1995. Twenty four answers that were sufficiently informative to be incorporated in the analysis were received. For your convenience we have tried to arrange and summarise the information in a summary for easy digestion. The circulation of 
the complete responses is restricted to those who have participated.

\section{Company data}

Companies have been divided in four groups: chemical, oil, engineering and catalyst producer. The size of the companies, as reflected by total employees, varies more than one order of magnitude. This holds as well for the total research effort.

Also the number of researchers, active in heterogeneous catalysis shows a wide variation for the companies considered in this survey. A total of approximately 1600 researchers in heterogeneous catalysis works with the companies which responded. Oil companies employ $40 \%$ of these researchers, while chemical, engineering and catalyst producing companies employ resp. $30 \%, 20 \%$ and $10 \%$.

\section{Utilisation of kinetic data}

A summary of the results for all responding companies is given in Table 1 where average numbers as well as standard deviations are listed for the various company types as well as for the overall. Following observations can be made on the utilisation of kinetic data:

- There is sometimes a wide variation in utilisation among companies; e.g., trouble shooting by chemical companies $(14 \%$, s.d. $14 \%)$ or catalyst development by oil companies (24\%, s.d. $20 \%$ ).

- There are no significant differences between chemical and oil companies.
- As expected catalyst producers focus on catalyst development (47\% utilisation) while engineering companies concentrate on process development (55\% utilisation).

- The total data show that there are three (equally important) utilisation areas:

- Process development (31\%).

- Process optimisation (27\%).

- Catalyst development (26\%).

\section{Approach}

\subsection{Experimental approaches}

Nine respondents mention the use of external data, but only one relies exclusively on external sources. Industrial plants are explicitly reported as a data source by five companies.

The use of pilot plants is well spread, but there is a tendency towards down-scaling. Pilot plants are usually aimed at simulating directly the industrial operation although the difficulty to reproduce the hydrodynamic regimes are recognised. The synthesis of a sufficient amount of products, the effects of recycle streams and catalyst deactivation are cited as specific reasons for pilot plant studies.

Most of the responding companies (with one exception) make use of own test units to determine relations between process conditions and conversions and selectivities. These units are primarily designed for development and optimisation and consist usually of a continuously fed tubular reactor with up to $100 \mathrm{~g}$ of catalyst present as a fixed bed.

Table 1

Utilization of kinetic data (average by company type)

\begin{tabular}{|c|c|c|c|c|c|c|c|}
\hline Company type & $\begin{array}{l}\text { Number of } \\
\text { companies }\end{array}$ & $\begin{array}{l}\text { Trouble } \\
\text { shooting (\%) }\end{array}$ & $\begin{array}{l}\text { Process } \\
\text { development (\%) }\end{array}$ & $\begin{array}{l}\text { Process } \\
\text { optim. (\%) }\end{array}$ & $\begin{array}{l}\text { Catalyst } \\
\text { development }(\%)\end{array}$ & $\begin{array}{l}\text { Mechan. } \\
\text { research }(\%)\end{array}$ & Other \\
\hline Chemical & 8 & $14(14)$ & $29(7)$ & $27(9)$ & $22(9)$ & $7(8)$ & $1(-)$ \\
\hline Oil & 7 & $10(6)$ & $27(17)$ & $33(17)$ & $24(20)$ & $2(4)$ & $4(-)$ \\
\hline Catalyst producer & 4 & $16(17)$ & $13(5)$ & $14(8)$ & $47(17)$ & $10(12)$ & $0(-)$ \\
\hline Engineering & 5 & $2(3)$ & $55(19)$ & $27(7)$ & $15(10)$ & $1(2)$ & $0(-)$ \\
\hline Overall & 24 & $10(11)$ & $31(19)$ & $27(12)$ & $26(17)$ & $5(7)$ & $1(-)$ \\
\hline
\end{tabular}

Standard deviation is given in parentheses. 
Plug flow is commonly assumed. The isothermicity of the bed is not always ensured. The use of appropriate reactor models allowing to take into account the existence of temperature non-uniformity on bed scale is explicitly mentioned by one company. The usefulness of experiments at low feed conversions is reported by four respondents. Six companies acknowledge the need to perform experiments with industrial feedstocks and/or with feeds including recycle components.

Gradientless continuous flow reactors, mostly of the Berty-type, are or have been used by at least six companies.

The application of temperature programmed techniques is mentioned three times. One company also reports the use of isotopic labelling and so-called Temporal Analysis of Products (TAP).

There is no common practice concerning the nature of the collected data, i.e., whether the latter should basically be determined by chemical phenomena alone or not. The advantages of intrinsic kinetics are generally recognised but are not always considered to outweigh the difficulties to be overcome in obtaining them.

In short range projects or when complex feedstocks are involved apparent kinetics are considered appropriate. Two companies consider this is also the case when the hydrodynamics are well known, e.g., for gas flow over a fixed catalyst bed. Engineering companies are the most in favour of intrinsic kinetics, catalyst manufacturers the least. The latter are the most eager to perform experiments in set-ups similar to those on industrial scale and report the use of a broad scala of laboratory reactors from slurry reactors to microrisers. The separate study of hydrodynamics with cold flow experiments is mentioned once.

\subsection{Modelling approaches}

With respect to the modelling approaches, we will distinguish between the different types of the kinetic model itself (say 'rate expression'):

- Type 1 - Simple first or $n$th order.

- Type 2 - Langmuir-Hinshelwood/Hougen-Watson/(LHHW).

- Type 3 - n-lump models (for complex mixtures).

- Type 4-Detailed mechanistic.

- Type 5-ab initio.
However, in addition we will distinguish between three different levels of separation of the 'intrinsic' reaction kinetics and transport phenomena:

- Level 1 - Intrinsic kinetic models, i.e.. excluding both internal and external heat- and mass transfer and mixing effects.

- Level 2 - Apparent kinetic models, which include internal transfer effects, but excluding external transfer and mixing effects.

- Level 3 - Models that include all transport/mixing effects in the 'kinetics'.

We make this level distinction, because it appeared that quite a number of respondents indicated the use of level 2 or 3 in combination with "more complex" kinetic models, type $>1$.

\subsection{Separation between intrinsic kinetics and transport phenomena}

Most of the respondents state that 'in principle' one should strive for intrinsic kinetics, but the majority nevertheless do (either sometimes or frequently) use level 2 or 3 instead, for various reasons. Constraints of time and funding are very important in this respect. In particular the catalyst manufacturers and contractor/engineering companies, but also some oil companies state that they mainly or frequently use level 3 models. Level 2 separation between kinetics/transport phenomena, i.e.. neglecting intraparticle diffusion, seems also widespread and explicitly mentioned by three companies. Nine respondents have indicated that they mainly use intrinsic kinetics. Some companies that apply (mostly) level 2 or 3 mention that they are planning (more) use of level 1 approaches.

\subsection{Kinetic model complexity}

Roughly half of the respondents state or indicate that they use (almost) all ranges of models, which are tailored to the application. Problems related to type 3 kinetic models are mentioned by two respondents.

The most common philosophy is 'as simple as possible', however, without lumping of (external) transfer resistances and mixing effects, i.e., level 1 or 2 . This is explicitly mentioned by eight respondents. Usually this leads to either $n$th order or ( sim- 
plified) LHHW models. Five respondents state that they use mostly LHHW models.

One company states that they usually start with simplified LHHW models and slowly build up complexity, if required. However, they also warn for the pitfall of using too many kinetic parameters, and therefore they advise not to use the approach of deriving and testing (a variety of) detailed mechanistic models. Another, however, sketches as ideal approach that a detailed elementary reaction mechanism is set-up and all corresponding parameters are determined, but they indicate to adopt scarcely this 'ideal' route.

Development of truly mechanistic or ab initio kinetic models are scarcely explicitly mentioned and applied only if there is really a high incentive.

\subsection{Miscellaneous}

Adopting models from other sources (literature, suppliers) is occasionally mentioned.

Some respondents have reflected on the applicability of the different modelling approaches with respect to scale-up. One respondent pointed out that for scaleup purposes sometimes it might be 'better' to use lumped kinetic models (provided hydrodynamic similarity is maintained). Closely related to this, another respondent mentions that validation of reactor/kinetic models against pilot-plant data has shown these to be rather unreliable.

The use of modern kinetic modelling/parameters optimisation software is reported by roughly half of the respondents. SimuSolv is mentioned most frequently. A limited number of in-house tools has been developed.

The use of experimental design as tool in model development is indicated by three companies. $\mathrm{Ab}$ initio methods as starting point for the construction of kinetic expressions are referred to only twice.

Advanced reactor modelling (including CFD) is mentioned by two respondents.

\section{Opportunities for improvement}

A number of points were addressed by companies from all four branches included in this questionnaire.

\subsection{Cost and time}

The most important problem is the fact that the determination of reliable kinetic data is too expensive and too time consuming, this being subscribed by almost all parties. Kinetic data are frequently missing even for existing processes. The following additional remarks were made in connection with cost and time:

- Apparatus is costly, not commercially available or not always accessible. Apparatus is claimed to be expensive because of high pressure needed, safety regulations, corrosive chemicals, sampling problems. high temperatures $\left(650^{\circ} \mathrm{C}\right.$ and above), or demanding conditions in general.

- Optimisation of experimental programs is troublesome.

- Very fast reactions, e.g., in the case of high temperatures, are challenging.

- Analytical problems are important, e.g.. with multi-component product mixtures.

- High temperature processes and homogeneous reactions are difficult to cover.

- Literature data are incomplete and/or unreliable, not applicable for relevant conditions or simply missing.

- Cost could be decreased by down-scaling in the case of trickle phase processes, if this were technically feasible.

\subsection{Deactivation}

The problems of deactivating catalysts and catalysts loosing selectivity are mentioned by many parties. Comparable complications are experienced for processes that are unstable because of other reasons then catalyst deactivation, e.g., oscillating processes. Both the experimental problem to determine the catalyst stability as well as the lack of suitable models are reported to be serious problems. Experimental problems include the fact that in many cases the determination of the relation between deactivation and its cause, e.g., coke formation, cannot be performed in situ.

It is claimed that successful modelling of a deactivating system has been based so far only on plant data. 
Deactivation of catalysts contributes significantly to the high costs for the determination of kinetic data.

\subsection{Modelling aspects}

The third well acknowledged blind spot is apparently the availability of modelling tools that are sufficiently user friendly. Modelling tools are defined here as all software and hardware needed to fit experimental data to a reaction scheme, as well as tools needed to predict the performance of a reactor based on kinetic data and calculation of mass and heat transfer. Many more specific wishes are formulated:

- Integration of the modelling of kinetics and heat/ mass transfer in an early stage of the development of a model, or integration of kinetic models with 3D computational fluid dynamics.

- 'Foolproof' regression methods.

- Make available a database with thermodynamic data and physical data, e.g., adsorption-coefficients, needed for the construction of models.

- A concept for the combination of heterogeneous and homogeneous reactions.

- The hope is expressed that molecular modelling will be increasingly helpful in the selection of reaction schemes.

- Modelling tools for novel structured catalytic reactors, e.g., monoliths and membranes as well as particular shaped catalysts.

\subsection{Additional remarks}

- Several respondents remark that additional problems occur in processes that are more complicated than the case of a solid catalyst applied in gas-phase processes. It is experienced by at least two companies that the fundamentals of catalysis in the liquid phase are desperately missing. The interaction of the catalyst (active phase, support) with, e.g., the solvent (or combination of solvents) is obscure and experimental techniques to study these phenomena are missing. One catalyst producer adds that a satisfying approach is missing for the determination of intrinsic kinetics in tricklephase processes. In addition, an engineering company reports that in some cases (e.g., fluid-bed reactors and three-phase-reactors) the hydrodynamics are the real bottleneck. A chemical company mentions that scale up of multiphase reactors needs improvement.

- Furthermore, problems caused by multi-component feeds and/or product mixtures, including impurities, are described by three respondents: how to measure kinetics and how to construct models in such a case?

- The fact that a catalyst may not be available in the form to be used in the final process may hamper the determination of the kinetics of a new process. The ill definition of commercial catalysts is sometimes a problem that cannot be solved yet.

- Isothermicity is often not ensured in laboratory reactors in the case of a exothermic reaction.

It is clear that the problems that are being described by chemical companies, oil companies and engineering contractors are not essentially different. Obviously the problems that are encountered reflect the business of the company (e.g., multi-component related problems are mentioned by oil companies and contractors active in oil processing). Nevertheless, the underlying causes of the problems are more or less similar.

\section{Future}

Several bottlenecks in the efficient utilisation of kinetics emerge from the answers. Their solution is certainly a major task. This is also reflected by the thoughts presented by the responding professionals. In most cases only routes to come to solutions are suggested, which are summarised below.

Many participants expect modelling, or even ab initio modelling, to become increasingly important, e.g., to determine the most likely reaction pathway. However, nobody is confident at which pace this development is likely to proceed.

Cooperation appears to be the magic word. There is general consensus that cooperation between industry and academia is necessary to solve the problems that have been indicated. The cooperation between the fundamental catalysis groups with groups working in the field of applied catalysis and reaction engineering should be improved as well. The methods suggested to arrive at the collaboration that is asked for are numerous: European Commission-type projects, projects explicitly not via the EC, bilateral contacts, consortia, mobility networks, exchange programs, 
contract research at consulting companies or academic facilities, Working Groups consisting of both academic and industrial researchers, etc.

Obviously, all the topics indicated in this report may be subject of cooperative research projects. However, some specific suggestions were made:

- Modelling and experimental techniques for G-L-S catalytic systems.

- Determine the kinetics of commercial processes in a world-wide cooperation and focus first on analytical problems, or construction of a general kinetic data-base.
- Development of automated laboratory reactors to determine intrinsic kinetics under demanding conditions, and definition of a toolkit of experimental techniques for all cases that may occur.

- Development of easy to handle software for fitting of kinetic models, by try-outs on a number of commercial systems, in order to arrive at a generally applicable system.

- Development of in situ methods to determine the real concentration of reactant on or close to the catalytic site, especially when mass transfer limitation cannot be circumvented. 